\title{
Kühlsauger \& Co: Performative Fremdsprachendidaktik in technischen Studiengängen
}

\section{Stefanie Giebert}

Im Workshop ging es um die Vorstellung von performativen Elementen im DaF-Unterricht, wie ich sie derzeit in meinen Kursen am Studienkolleg an der Hochschule Konstanz einsetze und weiterentwickle. Es handelt sich also um work in progress.

Die Ausgangssituation: An Studienkollegs bereiten sich junge Menschen aus nicht-EU-Ländern innerhalb eines Jahres auf ein Studium im Deutschland vor. Ich unterrichte im sogenannten T-Kurs, der auf ein technisches Studium vorbereitet. Dort unterrichte ich Deutsch mit je vier Wochenstunden im ersten und zweiten Semester.

Im Workshop stellte ich zu Anfang diese Ausgangssituation und die damit verbundenen spezifischen Herausforderungen für den Einsatz von Dramapädagogik dar. Erstens bestehen die Kurse aus großen, heterogenen Gruppen von 25-30 Lernenden. Zweitens sind da die Erwartungshaltungen der Lernenden: kreative und spielerische Unterrichtselemente sind für diese oft eher ungewohnt bzw. passen teilweise nicht zu ihren Vorstellungen von sinnvollem Unterricht. ${ }^{1}$ Drittens soll der Kurs auf eine Abschlussprüfung vorbereiten, in der akademisches Deutsch und Sachtexte im Vordergrund stehen, eine inhaltliche Ausrichtung, bei der ein dramapädagogisches Arbeiten vielleicht nicht das Naheliegendste ist. ${ }^{2}$ Die erste Herausforderung dürfte bildungsgang- und länderübergreifend von vielen Lehrenden ähnlich erlebt werden, die anderen beiden sind eher spezifisch für diesen Kurs, lassen sich aber leichter angehen. Einerseits versuche ich, die spielerischen und performativen Elemente in Mikroform (z.B. Vokabelraten mit Erklären, Zeichnen, Pantomime à la Activity) einzuführen und dann langsam auszuweiten: zum Beispiel mit einem Gallery Walk (Erfindermesse), der Aufführung einer kurzen Szene in einer Stunde (Murks \& Co.), bis hin zu einem größeren Szenenprojekt (die Ingenieursserie) am Ende des ersten Semesters. Meine Hoffnung ist, dass durch langsame Gewöhnung und wiederholte Begegnung mit performativen Formen

\footnotetext{
${ }^{1}$ Eine gewisse Skepsis äußert sich dann recht deutlich in Kommentaren wie: „Das ist doch Schrott, ich meine, das ist doch blöd.“ oder „Das geht nicht.“ bzw. „Jetzt ist aber erst mal genug Kreativität" (sinngemäß wiedergegebene Kommentare dreier Studierender am Anfang bzw. Ende einer dramapädagogischen Einheit im WS 2017/18 und WS 18/19).

2 Die sogenannte Feststellungsprüfung entspricht der DSH (Deutsche Sprachprüfung für den Hochschulzugang).
} 
die Skepsis der Studierenden nachlässt und sie das Performative als normalen Teil des Deutschunterrichts wahrnehmen. Was die dritte Herausforderung angeht, so kann akademisches Deutsch und Sachtexte durchaus in performative Aktivitäten eingebunden werden.

Nach Kennenlernen des Kontextes sollten die Workshopteilnehmer einige der dramapädagogischen Übungen, nämlich eine Aufwärmübung und eine sprachfokussierte Übung (Erfindermesse) selbst ausprobieren. Ein Ausprobieren der weiteren Elemente (szenische Umsetzung eines Sachtextes und Entwicklung einer Szene zum Thema „Serie über Ingenieure") hätte den Rahmen des Workshops gesprengt. Abschließend war eine Diskussionsrunde geplant, die jedoch aus Zeitgründen etwas kurz ausfiel. Im Folgenden möchte ich die vorgestellten und ausprobierten Übungen kurz beschreiben: Nach kurzem Antesten des Vokabelratespiels (das den Teilnehmern des Workshops bekannt war), folgte eine Aufwärmübung aus dem Improtheater zum Blockieren und Annehmen von Ideen („yes, but...“ vs. „yes, and..."). Bei dieser Paarübung macht Person A fortlaufend Vorschläge zu einem Thema (z. B. Pläne für den Abend), während Person B ständig Einwände vorbringt. In der zweiten Runde macht wiederum A Vorschläge. Nun akzeptiert B und ergänzt um eigene, dazu passende Ideen. Frage ich Studierende, wie sie den Unterschied zwischen den beiden Modi wahrnehmen, kommt häufig die Antwort, dass die erste Variante eher anstrengend und frustrierend sei, während die zweite sich angenehmer und produktiver anfühle. ${ }^{3}$ Auf diese Weise hoffe ich, den Studierenden zu vermitteln, dass Teamwork bei einer gewissen Aufgeschlossenheit einfach besser funktioniert.

Die zweite Übung setze ich in einer Einheit zum Thema „Ingenieure und Erfindungen" ein. Die Studierenden haben sich vorher mit den sprachlichen Strukturen von Definitionen und mit Funktionsbeschreibungen im Passiv beschäftigt. Wie auch die Lernenden erhielten die Teilnehmerinnen des Workshops Kärtchen mit einem Wortteil, der Hälfte eines Gerätes (z. B. „Kühl-“ oder „-sauger"). Im Unterricht lasse ich normalerweise kurz die „richtigen“ Wortpaare finden und schreibe sie an. Danach sollen die Studierenden als „Erfinder" tätig werden und sich einen Partner suchen, mit dem sich eine ganz neue Maschine ergibt und diese dann definieren, beschreiben und schließlich mit einem Poster der Klasse vorstellen. ${ }^{4}$. Dabei soll das Gerät auf einer Art Bauplan dargestellt und mit kurzen Sätzen im Passiv in seiner Funktion beschrieben werden. Angaben zu Bauteilen, Verkaufspreis und intendierter Zielgruppe sind ebenfalls willkommen. Anschließend halten die Studierenden bzw. hielten die Teilnehmerinnen des Workshops eine kurze Präsentation zu ihrem Gerät. Im

\footnotetext{
${ }^{3}$ Manchmal kommt jedoch auch die Antwort, dass es Spaß mache, alles verneinen zu dürfen und den anderen ins Schwitzen zu bringen.

${ }^{4}$ Dies ist erfahrungsgemäß der Moment, wo sich Widerstand regt und manche Studierende sich zuerst einen Partner suchen, mit dem sich ein tatsächlich existierendes Gerät bilden lässt. Wenn ich dann klarmache, dass die Aufgabe ist, wirklich eine neue Maschine zu „erfinden“, kommt von manchen Studierenden eine ablehnende Reaktion und sie gehen mit einer gewissen Skepsis an die Arbeit. Andere Studierende sind hingegen sofort begeistert bei der Sache und entwerfen ihre Maschine.
} 
Unterricht läuft dies so ab, dass ca. die Hälfte der Gruppe präsentiert, während die andere das Publikum bildet und von einer Erfindung zur nächsten schlendert und auch Fragen an die „Erfinder“ stellt (danach Wechsel). Idealerweise sind bei dieser Form alle Lernenden beschäftigt. Die Präsentierenden können ihre Vorträge ohne großen Druck (sie sprechen nur vor einer Kleingruppe) und auch mehrfach halten. Die Beteiligten kommen durch die Nachfragen ins Gespräch, sodass nicht nur Darstellung von Vorbereitetem gefragt ist, sondern auch spontanes Improvisieren. Wenn die Übung gut läuft, ist die Stimmung im Kurs positiv und aufgelockert, es wird über die kuriosen Maschinen gelacht und idealerweise sind alle präsentierend oder fragend involviert. ${ }^{5}$ Um die so ,performten' sprachlichen Elemente noch einmal zu vertiefen, füllen die Lernenden als Hausaufgabe ein Arbeitsblatt zur Produktbeschreibung aus. Gerne hätte ich im Workshop noch über diese Übung diskutiert und ihr Feedback dazu gehört, allerdings ließ das der Zeitrahmen dann doch nicht mehr zu. ${ }^{6}$

Zum Abschluss meines Beitrags möchte ich noch noch über den Begriff ,performativ' bzw. ,performance‘ reflektieren. Mein Verständnis dieses Begriffs hat sich durch die Tagung eigentlich nicht erweitert, da es von vornherein weit gefasst war, ich sah mich eher bestätigt. Ich sehe ,performativ' im Unterricht immer als zweiteilig. Einerseits die ,performativen' Übungen, bei denen die Lernenden vor einem wie auch immer gearteten Publikum etwas tun. Andererseits die Rolle des Lehrenden als ,performer'. Interessant für mich war in meinem Workshop, dass ich das Gefühl hatte, ich müsse die Teilnehmerinnen durch meine ,performance' nach einem bereits langen Tag erst einmal wieder aktivieren. Es war Spätnachmittag und der Workshopstart hatte sich verzögert und ich nahm eine gewisse Erschöpfung wahr, sobald sie jedoch selbst aktiv werden konnten, schienen die Energielevels wieder zu steigen. Im Unterrichtsalltag begegnen mir ebenfalls häufig müde Lernende, dazu kommt bei manchen ein gewisses Desinteresse am Fach. Es wäre sicher sinnvoll, bei einer müden Klasse spontan eine kurzes spielerisches und/oder performatives Element einschieben zu können, jedoch ohne den eigenen Stoffplan durcheinander zu bringen oder sich den Vorwurf besonders ehrgeiziger Studierender einzuhandeln, dass man in dieser Zeit nichts lerne hier arbeite ich noch an meiner eigenen Flexibilität.

Schließlich noch zur Situation an meiner Fachhochschule: Es gibt dort sicher Akteure, die performatives Lernen praktizieren - von Architekturstudenten, die Werkschauen abhalten, bis zu Wirtschaftswissenschaftlern, die Planspiele durchführen (in den technischen Fächern kann ich die Situation schwer einschätzen). In meiner Wahrnehmung habe ich jedoch als Sprachdozentin

\footnotetext{
${ }^{5}$ Bei großen Gruppen besteht allerdings auch die Gefahr, dass Studierende nur einmal präsentieren und dann denken, sie seien jetzt ja ,fertig' und könnten sich nun wieder ihrem Smartphone widmen - hier interveniere ich meistens und bringe ihnen neues Publikum.

${ }^{6}$ Die Zeit ist meiner Erfahrung nach eine weitere Herausforderung im Unterrichtsalltag, da dramapädagogische Elemente oft zeitintensiv sind. Ich als Lehrende kann Studierende zwar zur Eile antreiben, finde das aber gerade bei Übungen, die Kreativität erfordern, nicht immer angemessen.
} 
wenig Einfluss, von daher sehe ich mich außerhalb meiner eigenen Praxis und einer jährlich von mir in Zusammenarbeit mit einer Schweizer Kollegin organisierten Tagung, die aber fast nur von externen Teilnehmern besucht wird, in keiner geeigneten Position, das performative Lehren, Lernen und Forschen dort zu auszubauen. 